\title{
Maxbias curves of robust scale estimators based on subranges
}

\author{
Christophe Croux $^{1}$, Gentiane Haesbroeck ${ }^{2}$ \\ ${ }^{1}$ ECARES, Université Libre de Bruxelles, CP-114, Av. F.D. Roosevelt 50, B-1050 Brussels, \\ Belgium. (e-mail: ccroux@ulb.ac.be) \\ ${ }^{2}$ Department of Economics, University of Liège, Boulevard du Rectorat 7, B-4000 Liège, \\ Belgium. (e-mail: g.haesbroeck@ulg.ac.be)
}

Received: February 2000

\begin{abstract}
A maxbias curve is a powerful tool to describe the robustness of an estimator. It is an asymptotic concept which tells how much an estimator can change due to a given fraction of contamination. In this paper, maxbias curves are computed for some univariate scale estimators based on subranges: trimmed standard deviations, interquantile ranges and the univariate Minimum Volume Ellipsoid (MVE) and Minimum Covariance Determinant (MCD) scale estimators. These estimators are intuitively appealing and easy to calculate. Since the bias behavior of scale estimators may differ depending on the type of contamination (outliers or inliers), expressions for both explosion and implosion maxbias curves are given. On the basis of robustness and efficiency arguments, the MCD scale estimator with $25 \%$ breakdown point can be recommended for practical use.
\end{abstract}

Key words: Breakdown point, Maxbias Curve, Robustness, Scale Estimation

AMS subject Classifications: 62F35, 62G35

\section{Introduction}

Scale estimators are frequently used in data analysis: they measure dispersion and are needed to standardize data or residuals. They are also used to check for outlying observations, e.g. in quality control. The most popular scale estimator is the standard deviation, which is however known to be extremely sensitive to outliers. Therefore, it is often replaced by the interquartile range

$$
\mathrm{IQR}_{n}=\left|x_{(n-\lfloor n / 4\rfloor)}-x_{(\lfloor n / 4\rfloor+1)}\right|
$$

where $x_{(1)} \leq x_{(2)} \leq \cdots \leq x_{(n)}$ are the sorted observations and $\lfloor z\rfloor$ denotes the 
largest integer smaller than or equal to $z$. A more general version of the IQR is the $\alpha$-range, which is defined as

$$
R_{n, \alpha}=c_{\alpha}\left|x_{(n-\lfloor\alpha n\rfloor)}-x_{(\lfloor\alpha n\rfloor+1)}\right| .
$$

The trimming proportion equals thus (approximately) $2 \alpha$, with $0<\alpha<\frac{1}{2}$. The constant $c_{\alpha}$ will guarantee consistency of the estimator at the chosen model distribution. The limit case $\alpha=0$ yields the ordinary (non robust) range, while $\alpha=0.25$ corresponds to the interquartile range. As an associated measure of location, the midpoint of the interval $I_{\alpha}=\left[x_{(\lfloor\alpha n\rfloor+1)} ; x_{(n-\lfloor\alpha n\rfloor)}\right]$ can be taken, yielding the $\alpha$-midrange

$$
M_{n, \alpha}=\frac{1}{2}\left(x_{(\lfloor\alpha n\rfloor+1)}+x_{(n-\lfloor\alpha n\rfloor)}\right) .
$$

Instead of taking the middle and length of the interval $I_{\alpha}$ as location and scale measures, one could also compute mean and standard deviation over this interval. Then the $\alpha$-trimmed mean

$$
T_{n, \alpha}=\frac{1}{n-2\lfloor n \alpha\rfloor} \sum_{i=\lfloor\alpha n\rfloor+1}^{n-\lfloor\alpha n\rfloor} x_{(i)}
$$

and the $\alpha$-trimmed standard deviation

$$
S_{n, \alpha}=\sqrt{\frac{d_{\alpha}}{n-2\lfloor n \alpha\rfloor} \sum_{i=\lfloor\alpha n\rfloor+1}^{n-\lfloor\alpha n\rfloor}\left(x_{(i)}-T_{n, \alpha}\right)^{2}}
$$

are obtained, where $d_{\alpha}$ is a consistency factor and $0<\alpha<\frac{1}{2}$. The choice $\alpha=0$ yields the usual mean and standard deviation.

The previous location and scale estimators rely on the interval $I_{\alpha}$. Two other scale estimators will be considered as competitors for $R_{n, \alpha}$ and $S_{n, \alpha}$ : the univariate versions of the Minimum Volume Ellipsoid (MVE) estimator and of the Minimum Covariance Determinant (MCD) estimator. The MVE and MCD estimators were introduced by Rousseeuw (1985) and are routinely used for estimating robust covariance matrices. But also in a univariate setup they can be very useful. The MVE-scale estimator is, as $R_{n, \alpha}$, a range computed over an interval, while the MCD-scale estimator is, as $S_{n, \alpha}$, a standard deviation computed over a certain interval. Of course, these intervals will not necessarily coincide with $I_{\alpha}$.

The univariate MVE scale estimator is given by the length of the shortest interval containing at least a proportion $(1-\alpha)$ of the observations. More formally

$$
\operatorname{MVE}_{n, \alpha}=k_{\alpha} \min _{1 \leq i \leq n-\lfloor n(1-\alpha)\rfloor}\left|x_{(i+\lfloor n(1-\alpha)\rfloor)}-x_{(i)}\right|
$$

where $k_{\alpha}$ is again for consistency and $0<\alpha \leq \frac{1}{2}$. This scale estimator is often referred to as the shortest half (Rousseeuw and Leroy, 1988). The related location estimator is given by the center of the interval which corresponds to the minimum in (1.2). 
The univariate MCD estimator consists in considering all subsamples of $h$ points of $X=\left\{x_{1}, \ldots, x_{n}\right\}$, where $h=\lfloor n(1-\alpha)\rfloor+1$, and selecting that subsample which has the smallest value of the standard deviation computed over the subsample. The standard deviation of this optimal subsample serves as the MCD-scale estimator, while the location part of the MCD estimator is given by the average over the selected subsample. Since the more dispersed the $h$ points are, the bigger the standard deviation will be, the $h$ points determining MCD need to form a contiguous subsample of $X$. The finite-sample definition of the MCD-scale estimator can therefore be written as

$$
\mathrm{MCD}_{n, \alpha}^{2}=l_{\alpha} \min _{1 \leq i \leq n-\lfloor n(1-\alpha)\rfloor} \operatorname{Var}\left\{x_{(i)}, \ldots, x_{(i+\lfloor n(1-\alpha)\rfloor)}\right\}
$$

where $\operatorname{Var}\left\{x_{(i)}, \ldots, x_{(i+\lfloor n(1-\alpha)\rfloor)}\right\}$ is the variance over the subsample $\left\{x_{(i)}, \ldots\right.$,

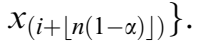

The $\mathrm{MVE}_{n, \alpha}$ and $\mathrm{MCD}_{n, \alpha}$ estimators are very simple to implement and are standard available in some software packages. Both estimators can be computed in $O(n \log n)$ (Rousseeuw and Leroy, 1987, page 172).

Subrange estimators are intuitively appealing and have already been studied extensively in the literature (Bickel and Lehmann 1976, Croux and Rousseeuw 1992). In this paper their maxbias curves will be computed and compared. A maxbias curve gives the maximal bias that an estimator can suffer from when a proportion $\varepsilon$ of the data come from a contaminated distribution (while the other $(1-\varepsilon) \times 100 \%$ of the data follow the model distribution). By letting $\varepsilon$ vary between zero and the breakdown point (which is the highest fraction of contamination that an estimator can withstand before becoming degenerate) a curve is obtained. Martin and Zamar (1993) already computed maxbias curves for other classes of scale estimators. This paper complements their work.

In Section 2, the maxbias curve is defined and functional representations of the considered estimators are given. Section 2 also contains the mathematical expressions for the maxbias curves and introduces the concept of a "regular exploding" maxbias curve. The different estimators are then compared in Section 3 with respect to their robustness and efficiency properties. Especially the MCD-scale estimator with $\alpha=0.25$ appears to have good properties, partly thanks to its non regular exploding maxbias curve. The last section draws some conclusions.

\section{Maxbias curves}

\subsection{Definition}

There exist several measures of robustness of an estimator (cfr. Hampel et al. 1986), but in this paper the maxbias curve will be used. The maxbias curve is a rich tool that summarizes several aspects of the robustness of an estimator, including the breakdown point and gross-error sensitivity. A survey on maxbias curves is given by Rousseeuw (1998).

The distribution that generates the good observations belongs to a location-scale family 


$$
\left\{F_{\mu, \sigma}(x):=F\left(\frac{x-\mu}{\sigma}\right) \mid 0<\sigma<\infty,-\infty<\mu<\infty\right\} .
$$

Throughout the paper, the central model distribution $F$ is supposed to satisfy the following property

$(F) F$ has a strictly positive and continuous unimodal density $f$ which is symmetric about the origin.

Let $S$ denote a statistical functional representing a scale estimator. All the scale functionals considered in this paper are affine equivariant (meaning that $S(a X+b)=|a| S(X)$ where $S(X) \equiv S(G)$ whenever $X \sim G)$ and Fisher consistent at the specified model, thus $S\left(F_{\mu, \sigma}\right)=\sigma$. Therefore there will be no loss of generality to restrict attention to the central model distribution $F$. Contamination may cause $S$ to become very large (explosion) or close to zero (implosion). Define the contamination neighborhood of $F$

$$
\mathscr{F}_{\varepsilon}=\{G ; G=(1-\varepsilon) F+\varepsilon H ; H \text { any distribution }\}
$$

for a given fraction of contamination $\varepsilon(0 \leq \varepsilon \leq 1)$.

The explosion maxbias curve of the scale estimator $S$ is now defined by

$$
B^{+}(\varepsilon ; S, F)=\sup _{G \in \mathscr{F}_{\varepsilon}} S(G)
$$

and the implosion maxbias curve of $S$ by

$$
B^{-}(\varepsilon ; S, F)=\inf _{G \in \mathscr{F}_{\varepsilon}} S(G) .
$$

Two important robustness measures can be derived from the maxbias curves. The asymptotic breakdown point

$$
\varepsilon^{*}(S, F)=\inf \left\{\varepsilon>0 \mid B^{+}(\varepsilon ; S, F)=\infty \text { or } B^{-}(\varepsilon ; S, F)=0\right\}
$$

which can be interpreted as the smallest proportion of bad points that are needed to let $S$ implode to zero or explode to infinity. One distinguishes furthermore between the explosion breakdown point $\varepsilon^{+}(S, F)=$ $\inf \left\{\varepsilon>0 \mid B^{+}(\varepsilon ; S, F)=\infty\right\}$ and the implosion breakdown point $\varepsilon^{-}(S, F)=$ $\inf \left\{\varepsilon>0 \mid B^{-}(\varepsilon ; S, F)=0\right\}$. Of course, $\varepsilon^{*}(S, F)=\min \left(\varepsilon^{+}(S, F), \varepsilon^{-}(S, F)\right)$. The sensitivity to small amounts of outliers can be measured by the gross-error sensitivity

$$
\gamma^{*}(S, F)=\max \left({\frac{\partial B^{+}(\varepsilon ; S, F)}{\partial \varepsilon}}_{\mid \varepsilon=0},-\frac{\partial B^{-}(\varepsilon ; S, F)}{\partial \varepsilon}\right) .
$$

Under some regularity conditions (He and Simpson 1993), $\gamma^{*}(S, F)$ equals as well the supremum of the absolute value of the influence function of $S$.

The derivation of the maxbias curve of a scale estimator is not always an easy matter. It is often admitted that the maximal explosion bias of a scale functional is produced by taking for $H$ in (2.1) a point mass at infinity while the highest implosion bias is due to a point mass placed at the center of the distribution. However, the most unfavorable or worst contaminating distribution $H$ is not necessarily of that type and in principle it doesn't even need to be 
a Dirac measure. Therefore it needs to be proven that the presumed worst contaminating distribution leads indeed to the supremum/infimum in (2.2) or (2.3). Expressions for the maxbias curves of the introduced scale estimators are given in the next two sections. The proofs of the propositions have been kept for the appendix, while the proofs of the lemmas can be found in Croux and Haesbroeck (1999b).

\subsection{Maxbias for the $\alpha$-range and $\alpha$-trimmed standard deviation}

To compute the maxbias curve the functional versions of the estimators have to be written down. The $\alpha$-range functional is defined for every distribution $G$ as

$$
R_{\alpha}(G)=c_{\alpha}\left|G^{-1}(1-\alpha)-G^{-1}(\alpha)\right|,
$$

where $G^{-1}(\beta)=\inf \{t \mid G(t)>\beta\} \quad$ for $\quad$ every $\quad 0 \leq \beta \leq 1 \quad$ and $\quad c_{\alpha}=$ $\left(2 F^{-1}(1-\alpha)\right)^{-1}$. Note that the value of the consistency factor depends on the chosen model.

Let us now turn to the $\alpha$-trimmed mean and standard deviation. The functional representation of the $\alpha$-trimmed mean is often given as

$$
T_{\alpha}(G)=\frac{1}{1-2 \alpha} \int_{G^{-1}(\alpha)}^{G^{-1}(1-\alpha)} y d G(y),
$$

where $0<\alpha<\frac{1}{2}$. A required property of a trimmed mean is affine equivariance, meaning that for all $a, b \in \mathbb{R}$,

$$
T_{\alpha}(a X+b)=a T_{\alpha}(X)+b,
$$

where $T_{\alpha}(X) \equiv T_{\alpha}(G)$ for $X \sim G$. A problem may arise when $G$ has atoms at the quantiles $G^{-1}(\alpha)$ or $G^{-1}(1-\alpha)$. In this case, the total mass of $G$ over the interval $\left[G^{-1}(\alpha), G^{-1}(1-\alpha)\right]$ may be bigger than $1-2 \alpha$ making relation $(2.5)$ fail. Therefore, definition (2.4) needs to be slightly adapted by adjusting the weights at the endpoints $G^{-1}(\alpha)$ and $G^{-1}(1-\alpha)$. Define

$$
\delta_{1}(G)=P_{G}\left(X \leq G^{-1}(\alpha)\right)-\alpha \quad \text { and } \quad \delta_{2}(G)=P_{G}\left(X \geq G^{-1}(1-\alpha)\right)-\alpha
$$

and the probability measure $G^{\alpha}$ by

$G^{\alpha}(\{A\})$

$=\frac{G\left(\{A \cap] G^{-1}(\alpha), G^{-1}(1-\alpha)[\}\right)+\delta_{1}(G) I\left(G^{-1}(\alpha) \in A\right)+\delta_{2}(G) I\left(G^{-1}(1-\alpha) \in A\right)}{1-2 \alpha}$

for every Borel measurable set $A$ (the indicator function $I(x \in A)$ equals 1 if $x$ belongs to $A$ and 0 if not). The distribution $G^{\alpha}$ can be seen as a restriction of $G$ to the interval $\left[G^{-1}(\alpha), G^{-1}(1-\alpha)\right]$. The functional definition of the $\alpha-$ trimmed mean which will be used in this paper is 


$$
T_{\alpha}(G)=E_{G^{\alpha}}[X] .
$$

It is easy to check that (2.8) satisfies (2.5). The quantities $\delta_{1}(G)$ and $\delta_{2}(G)$ only matter in the peculiar case when $G$ has atoms at the endpoints of $\left[G^{-1}(\alpha), G^{-1}(1-\alpha)\right]$. Otherwise, (2.8) equals (2.4). Similarly, the $\alpha$-trimmed standard deviation functional $S_{\alpha}(G)$ is defined as

$$
S_{\alpha}^{2}(G)=d_{\alpha} \operatorname{Var}_{G^{\alpha}}[X],
$$

where $d_{\alpha}=(1-2 \alpha) / \int_{\alpha}^{1-\alpha}\left(F^{-1}(t)\right)^{2} d t$.

Other widely used definitions for the $\alpha$-trimmed mean and standard deviation are

$$
\begin{aligned}
& T_{\alpha}(G)=\frac{1}{1-2 \alpha} \int_{\alpha}^{1-\alpha} G^{-1}(t) d t \\
& S_{\alpha}^{2}(G)=\frac{d_{\alpha}}{1-2 \alpha} \int_{\alpha}^{1-\alpha}\left(G^{-1}(t)-T_{\alpha}(G)\right)^{2} d t .
\end{aligned}
$$

These definitions can be shown to be equivalent to (2.8) and (2.9), but appeared to be more difficult to manipulate when making the proofs.

The derivation of the maxbias curves of the $\alpha$-range and $\alpha$-trimmed standard deviation both rely on the following two lemmas.

Lemma 1. Let $0 \leq \varepsilon<\alpha$ and $G=(1-\varepsilon) F+\varepsilon H$ where $F$ is the model distribution and $H$ an arbitrary distribution function and let $\Delta_{z}$ denote the Dirac measure which has all its mass at $z \in \mathbb{R}$. Then there exists a distribution $\tilde{H}=p_{1} \Delta_{G^{-1}(\alpha)}+p_{2} \Delta_{G^{-1}(1-\alpha)}$ with $p_{1}+p_{2}=1$ and $p_{1}, p_{2} \geq 0$ such that, with $\tilde{G}=(1-\varepsilon) F+\varepsilon \tilde{H}$,

(a) $G^{-1}(\alpha)=\tilde{G}^{-1}(\alpha)$ and $G^{-1}(1-\alpha)=\tilde{G}^{-1}(1-\alpha)$.

(b) $R_{\alpha}(G)=R_{\alpha}(\tilde{G})$ and $S_{\alpha}(G) \leq S_{\alpha}(\tilde{G})$.

This lemma essentially says that for any distribution $G$ belonging to the contamination neighborhood $\mathscr{F}_{\varepsilon}$, there exists a distribution $\tilde{G}$ consisting of a mixture of $F$ and two point masses which has a bias bigger than or equal to the bias of $G$, both for $R_{\alpha}$ and $S_{\alpha}$. Lemma 2 gives expressions which dominate $R_{\alpha}(\tilde{G})$ and $S_{\alpha}(\tilde{G})$.

Lemma 2. Let $0 \leq \varepsilon<\alpha$ and $G=(1-\varepsilon) F+\varepsilon H$ where $F$ is the model distribution and $H$ an arbitrary distribution function. Then there exists $\varepsilon_{1}$ with $0 \leq \varepsilon_{1} \leq \varepsilon$ such that

(a) $R_{\alpha}(G) \leq c_{\alpha}\left(F^{-1}\left(\frac{1-\alpha-\varepsilon_{1}}{1-\varepsilon}\right)-F^{-1}\left(\frac{\alpha-\varepsilon_{1}}{1-\varepsilon}\right)\right)$.

(b) $S_{\alpha}(G) \leq\left(\frac{d_{\alpha}(1-\varepsilon)}{1-2 \alpha} \int_{q_{1}\left(\varepsilon_{1}, \alpha\right)}^{q_{2}\left(\varepsilon_{1}, \alpha\right)}(y-\tilde{t})^{2} d F(y)\right)^{1 / 2}$ with $\tilde{t}=\frac{1-\varepsilon}{1-2 \alpha} \int_{q_{1}\left(\varepsilon_{1}, \alpha\right)}^{q_{2}\left(\varepsilon_{1}, \alpha\right)} y d F(y)$, where $q_{1}\left(\varepsilon_{1}, \alpha\right)=F^{-1}\left(\frac{\alpha-\varepsilon_{1}}{1-\varepsilon}\right)$ and $q_{2}\left(\varepsilon_{1}, \alpha\right)=F^{-1}\left(\frac{1-\alpha-\varepsilon_{1}}{1-\varepsilon}\right)$. 
The following propositions give expressions for the explosion and implosion maxbias curves of $R_{\alpha}$ and $S_{\alpha}$. Huber (1981, pages 125-126) already considered the bias behavior of both the $\alpha$-range and $\alpha$-trimmed standard deviation but only under symmetric contamination. Restricting $H$ in (2.1) to be symmetric seems to be an artificial condition, and leads to lower bias values.

Proposition 1. The explosion maxbias curve of the $\alpha$-range estimator at $F$ is given by

$$
B^{+}\left(\varepsilon ; R_{\alpha}, F\right)= \begin{cases}c_{\alpha}\left(F^{-1}\left(\frac{1-\alpha}{1-\varepsilon}\right)-F^{-1}\left(\frac{\alpha}{1-\varepsilon}\right)\right) & \text { if } \varepsilon<\alpha \\ \infty & \text { otherwise }\end{cases}
$$

and the implosion maxbias curve by

$$
B^{-}\left(\varepsilon ; R_{\alpha}, F\right)= \begin{cases}c_{\alpha}\left(F^{-1}\left(\frac{1-\alpha-\varepsilon}{1-\varepsilon}\right)-F^{-1}\left(\frac{\alpha}{1-\varepsilon}\right)\right) & \text { if } \varepsilon<1-2 \alpha \\ 0 & \text { otherwise }\end{cases}
$$

for $0<\alpha<\frac{1}{2}$.

Proposition 2. Let $q_{1}\left(\varepsilon_{1}, \alpha\right)=F^{-1}\left(\frac{\alpha-\varepsilon_{1}}{1-\varepsilon}\right)$ and $q_{2}\left(\varepsilon_{1}, \alpha\right)=F^{-1}\left(\frac{1-\alpha-\varepsilon_{1}}{1-\varepsilon}\right)$. Then the explosion maxbias curve of the $\alpha$-trimmed standard deviation estimator at $F$ is given by

$$
B^{+}\left(\varepsilon ; S_{\alpha}, F\right)= \begin{cases}\sqrt{\frac{d_{\alpha}(1-\varepsilon)}{(1-2 \alpha)} \int_{q_{1}(0, \alpha)}^{q_{2}(0, \alpha)}(y-t(\varepsilon))^{2} d F(y)} & \text { if } \varepsilon<\alpha \\ \infty & \text { if } \varepsilon>\alpha\end{cases}
$$

where $t(\varepsilon)=\frac{1-\varepsilon}{1-2 \alpha} \int_{q_{1}(0, \alpha)}^{q_{2}(0, \alpha)} y d F(y)$. The implosion maxbias curve is given by

$$
B^{-}\left(\varepsilon ; S_{\alpha}, F\right)= \begin{cases}\sqrt{\frac{d_{\alpha}(1-\varepsilon)}{1-2 \alpha} \int_{q_{1}(0, \alpha)}^{q_{2}(\varepsilon, \alpha)} y^{2} d F(y)} & \text { if } \varepsilon<1-2 \alpha \\ 0 & \text { otherwise }\end{cases}
$$

for $0<\alpha<\frac{1}{2}$.

It is often thought that maxbias curves always have a vertical asymptote at the breakdown point. Let us say that $B^{+}(\varepsilon ; S, F)$ has "regular" explosion if $\lim _{\varepsilon \uparrow \alpha} B^{+}(\varepsilon ; S, F)=\infty$, where $\alpha$ is the explosion breakdown point of $S$ at $F$. It follows now from the proof of Proposition 2 that, if the second moment of $F$ exists, $\lim _{\varepsilon \uparrow \alpha} B^{+}\left(\varepsilon ; S_{\alpha}, F\right)=B^{+}\left(\alpha ; S_{\alpha}, F\right)$ is finite and given by formula (2.14) for 
$\varepsilon=\alpha$. Therefore, the maxbias curve of the trimmed standard deviation has no regular explosion. It follows that the breakdown explosion rate $r^{+}$(Berrendero et al. 1998, 1999) of $S_{\alpha}$ with respect to any other $S$ having a maxbias curve with regular explosion and the same explosion breakdown point equals zero. That is

$$
r^{+}\left(S_{\alpha}, S\right)=\lim _{\varepsilon \uparrow \alpha} \frac{B^{+}\left(\varepsilon ; S_{\alpha}, F\right)}{B^{+}(\varepsilon ; S, F)}=0 .
$$

If the second moment of $F$ fails to exist, $B^{+}\left(\varepsilon ; S_{\alpha}, F\right)$ has again the typical "regular" explosion behavior.

\subsection{Maxbias for MVE and MCD scale estimators}

The MVE scale functional is defined as (cfr Rousseeuw and Leroy, 1988)

$$
\operatorname{MVE}_{\alpha}(G)=k_{\alpha} \inf _{0<t<\alpha}\left|G^{-1}(t+1-\alpha)-G^{-1}(t)\right|
$$

with $k_{\alpha}=\left(2 F^{-1}\left(1-\frac{\alpha}{2}\right)\right)^{-1}$. In (2.16), one considers intervals containing $(1-\alpha)$ of the mass of $G$. Such an interval takes the form $I_{G, x}=$ $\left[x-H_{G}(x), x+H_{G}(x)\right]$ where

$$
H_{G}(x)=\inf \left\{s>0 \mid P_{G}(|X-x| \leq s)>1-\alpha\right\} .
$$

An equivalent definition of $\mathrm{MVE}_{\alpha}$ which will be used in this paper, is then given by

$$
\operatorname{MVE}_{\alpha}(G)=k_{\alpha} \inf _{x}\left|I_{G, x}\right|=2 k_{\alpha} \inf _{x} H_{G}(x) .
$$

The optimal interval $I(G)$ has the smallest range among all the intervals $I_{G, x}$, so $I(G)=I_{G, x(G)}$ with $x(G)=\operatorname{argmin}_{x}\left|I_{G, x}\right|$. Note that $x(G)$ is the midpoint of the optimal interval $I(G)$ and equals the location part of the MVE functional.

The maxbias curve of the MVE scale estimator has already been derived by Martin and Zamar (1993). Before stating their result in Proposition 3, some notations must be introduced. Define for all $x \in \mathbb{R}, H_{\varepsilon}^{+}(x)$ as the solution $s$ of the equation

$$
F(x+s)-F(x-s)=\frac{1-\alpha}{1-\varepsilon}
$$

and $H_{\varepsilon}^{-}(x)$ as the solution $s$ of the equation

$$
F(x+s)-F(x-s)=\frac{1-\alpha-\varepsilon}{1-\varepsilon} .
$$


In particular,

$$
H_{\varepsilon}^{+}(0)=F^{-1}\left(\frac{2-\alpha-\varepsilon}{2(1-\varepsilon)}\right) \quad \text { and } \quad H_{\varepsilon}^{-}(0)=F^{-1}\left(\frac{2-\alpha-2 \varepsilon}{2(1-\varepsilon)}\right) .
$$

The form of the functions $H_{\varepsilon}^{+}$and $H_{\varepsilon}^{-}$is discussed in Hössjer et al. (1996).

Proposition 3. (Martin and Zamar, 1993) The explosion maxbias curve of the scale part of the $\mathrm{MVE}_{\alpha}$ estimator at $F$ is given by

$$
B^{+}\left(\varepsilon ; \mathrm{MVE}_{\alpha}, F\right)= \begin{cases}2 k_{\alpha} H_{\varepsilon}^{+}(0) & \text { if } \varepsilon<\alpha \\ \infty & \text { otherwise }\end{cases}
$$

and the implosion maxbias curve by

$$
B^{-}\left(\varepsilon ; \operatorname{MVE}_{\alpha}, F\right)= \begin{cases}2 k_{\alpha} H_{\varepsilon}^{-}(0) & \text { if } \varepsilon<1-\alpha \\ 0 & \text { otherwise }\end{cases}
$$

for $0<\alpha \leq \frac{1}{2}$.

The MCD-scale functional is defined analogously as the MVE, but now standard deviations instead of ranges are used. So,

$$
\begin{aligned}
& \operatorname{MCD}_{\alpha}^{2}(G) \\
& \quad=l_{\alpha} \inf _{0<t<\alpha}\left(\frac{1}{1-\alpha} \int_{t}^{t+1-\alpha}\left(G^{-1}(y)\right)^{2} d y-\left(\frac{1}{1-\alpha} \int_{t}^{t+1-\alpha} G^{-1}(y) d y\right)^{2}\right),
\end{aligned}
$$

where $l_{\alpha}=\left(2 \int_{0}^{F^{-1}(1-\alpha / 2)} y^{2} d F(y) /(1-\alpha)\right)^{-1}$.

Alternatively, $\mathrm{MCD}_{\alpha}^{2}$ is the minimal variance over all the intervals $I_{G, x}$, for $-\infty<x<\infty$. Denote again $I(G)$ the optimal interval and $x(G)$ its midpoint. It is known from the literature (Butler et al. 1993, Croux and Haesbroeck $1999 \mathrm{a})$ that at $I(G)$ (also called the MCD-solution), the average computed over that interval equals its midpoint $x(G)$. From the basic inequality $\operatorname{Var}(Z) \leq E\left[(Z-x)^{2}\right]$, for every random variable $Z$ and $x \in \mathbb{R}$, it follows that instead of minimizing the variance, one can as well minimize the squared deviation about the midpoint of the interval. Indeed, at the MCD-solution, the variance over $I(G)$ coincides with the squared deviation about $x(G)$, while for other values of $x$ the squared deviation over the interval $I_{G, x}$ about the midpoint $x$ is in any case bigger than or equal to the variance computed over $I_{G, x}$.

Therefore, a perfectly equivalent definition for the MCD functional is given by

$$
\operatorname{MCD}_{\alpha}^{2}(G)=l_{\alpha} \min _{x} \lambda_{G}(x)
$$

where

$$
\lambda_{G}(x)=\frac{1}{1-\alpha} \int_{x-H_{G}(x)}^{x+H_{G}(x)}(y-x)^{2} d G(x)+\frac{\delta_{G}(x) H_{G}(x)^{2}}{1-\alpha} .
$$


The integral in (2.26) is over an open interval. The mass at the endpoints has been corrected to have exactly mass equal to $1-\alpha$ over the interval $I_{G, x}$ by setting

$$
\delta_{G}(x)=1-\alpha-P_{G}\left(|x-X|<H_{G}(x)\right) .
$$

Of course, $\delta_{G}(x)$ equals zero for continuous distributions $G$. It appeared that definition (2.25) is much easier to handle when computing maxbias curves than definition (2.24). Moreover, $x(G)=\operatorname{argmin}_{x} \lambda_{G}(x)$ corresponds to the location part of the MCD functional.

Before deriving the maxbias curve of the MCD scale estimator, consider the following lemma which mainly relies on the unimodality property of the distribution function $F$.

Lemma 3. Let $F$ be a distribution function satisfying $(F)$. The function

$$
x \rightarrow \int_{x-H_{\varepsilon}^{+}(x)}^{x+H_{\varepsilon}^{+}(x)}(y-x)^{2} d F(y)
$$

increases strictly in $x$ towards infinity for $x \geq 0$. The same holds for $\mathrm{H}_{\varepsilon}^{+}$replaced by $\mathrm{H}_{\varepsilon}^{-}$.

Proposition 4. The explosion maxbias curve of the $\mathrm{MCD}_{\alpha}$ scale estimator at $F$ is given by

$$
B^{+}\left(\varepsilon ; \operatorname{MCD}_{\alpha}, F\right)= \begin{cases}\sqrt{\frac{l_{\alpha}(1-\varepsilon)}{1-\alpha} \int_{-H_{\varepsilon}^{+}(0)}^{H_{\varepsilon}^{+}(0)} y^{2} d F(y)} & \text { if } \varepsilon<\alpha \\ \infty & \text { if } \varepsilon>\alpha\end{cases}
$$

and the implosion maxbias curve by

$$
B^{-}\left(\varepsilon ; \operatorname{MCD}_{\alpha}, F\right)= \begin{cases}\sqrt{\frac{l_{\alpha}(1-\varepsilon)}{1-\alpha} \int_{-H_{\varepsilon}^{-}(0)}^{H_{\varepsilon}^{-}(0)} y^{2} d F(y)} & \text { if } \varepsilon<1-\alpha \\ 0 & \text { otherwise }\end{cases}
$$

for $0<\alpha \leq \frac{1}{2}$

Following the same reasoning as in the proof of Proposition 4 shows that $B^{+}\left(\alpha ; \mathrm{MCD}_{\alpha}, F\right)<\infty$ whenever the second moment of the distribution $F$ exists. Therefore, the explosion maxbias curve of $\mathrm{MCD}_{\alpha}$ has no regular explosion and will always be under that of $\mathrm{MVE}_{\alpha}$ in the neighborhood of the breakdown point. If the second moment of $F$ does not exist, then $\mathrm{MCD}_{\alpha}$ has again a maxbias curve with regular explosion.

As a Corollary of Propositions 1 to 4 , the breakdown points of the $\alpha$-range, $\alpha$-trimmed standard deviation, $\mathrm{MVE}_{\alpha}$ and $\mathrm{MCD}_{\alpha}$ are readily obtained.

Corollary. The asymptotic breakdown point of the estimators $R_{\alpha}$ and $S_{\alpha}$ at $F$ is given by 


$$
\varepsilon^{*}=\min (\alpha, 1-2 \alpha)
$$

while the asymptotic breakdown point of the estimators $\mathrm{MVE}_{\alpha}$ and $\mathrm{MCD}_{\alpha}$ at $F$ is equal to

$$
\varepsilon^{*}=\min (\alpha, 1-\alpha) .
$$

The maximal breakdown point for $R_{\alpha}$ and $S_{\alpha}$ equals $1 / 3$ and is obtained by choosing $\alpha=1 / 3$. For $\mathrm{MVE}_{\alpha}$ and $\mathrm{MCD}_{\alpha}$, the maximal breakdown point is $\varepsilon^{*}=1 / 2$ by taking $\alpha=1 / 2$. It is well-known that $1 / 2$ is also the upper bound of the breakdown point of any affine equivariant scale functional.

\section{Comparison of the bias behavior}

In this section, the bias behaviors of $R_{\alpha}, S_{\alpha}, \mathrm{MVE}_{\alpha}$ and $\mathrm{MCD}_{\alpha}$ are compared. For $F$, we will consider the Normal distribution, which satisfies condition $(\mathrm{F})$.

In Section 2, the effects of outliers and inliers on the bias of the estimator were measured by two separate curves, but both effects can be captured together by a global maxbias curve. Such a curve takes the form $g\left(B^{+}(\varepsilon ; S, F), B^{-}(\varepsilon ; S, F)\right)$ and is increasing in the first argument and decreasing in the second. It explodes whenever $B^{+}(\varepsilon ; S, F)$ explodes or $B^{-}(\varepsilon ; S, F)$ implodes. In this paper, two global maxbias curves will be computed: the logarithmic global maxbias curve proposed by Martin and Zamar (1989) which is defined as

$$
B_{1}(\varepsilon ; S, F)=\max \left(\ln \left(B^{+}(\varepsilon ; S, F)\right),-\ln \left(B^{-}(\varepsilon ; S, F)\right)\right),
$$

and the regression global maxbias curve given by

$$
B_{2}(\varepsilon ; S, F)=\sqrt{\left(\frac{B^{+}(\varepsilon ; S, F)}{B^{-}(\varepsilon ; S, F)}\right)^{2}-1} .
$$

The regression global maxbias curve gives information of the bias behavior of robust regression estimators based on the minimization of an affine equivariant scale measure $S$. Take the standard regression model $Y=\gamma+\beta^{t} X+\varepsilon$ with $\varepsilon \sim \mathrm{N}\left(0, \sigma^{2}\right)$ and define

$$
\hat{\beta}_{S}=\underset{\beta}{\operatorname{argmin}} S\left(Y-\gamma-\beta^{t} X\right) \text {. }
$$

It follows from arguments given by (Berrendero and Romo 1998) that, under some conditions on $S$, the maxbias curve of $\hat{\beta}_{S}$ is given by (3.2) if the carriers $X$ follow a multivariate normal distribution. If we take for $S$ the shortest half, then $\hat{\beta}_{S}$ is the Least Median of Squares $\left(\mathrm{LMS}_{\alpha}\right)$ estimator and $B_{2}\left(\varepsilon ; \mathrm{MVE}_{\alpha}, F\right)$ equals its maxbias curve. When $S$ is the $\mathrm{MCD}_{\alpha}$ estimator, the Least Trimmed Squares $\left(\mathrm{LTS}_{\alpha}\right)$ regression estimator is obtained. Maxbias curves for the LMS estimator were already obtained by Martin et al. (1989) and for LTS by Croux et al. (1996), but only for regression models without intercept.

In Figure 1, the explosion, implosion and global maxbias curves of the four scale estimators are represented for $\alpha=1 / 4$ at the Normal distribution. 


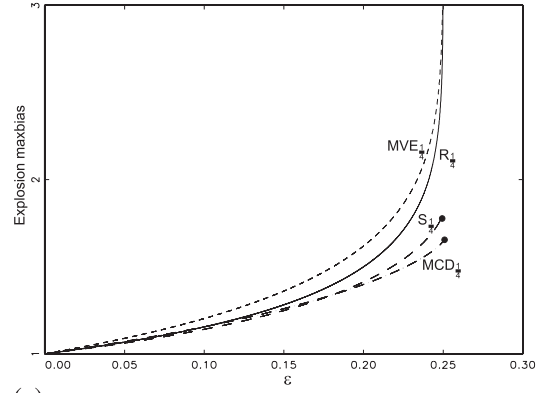

(a)

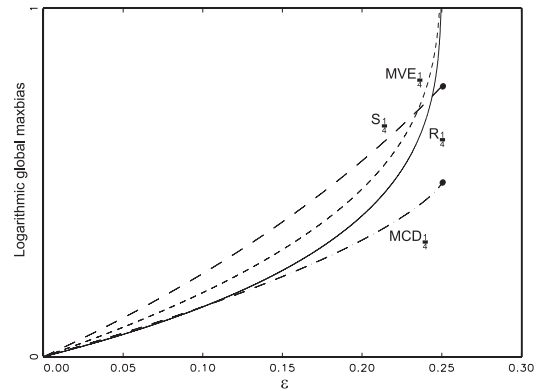

(c)

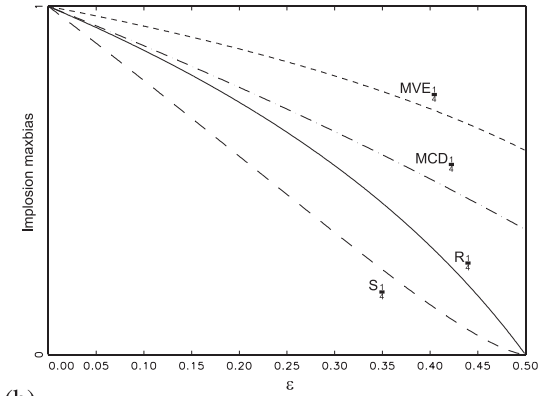

(b)

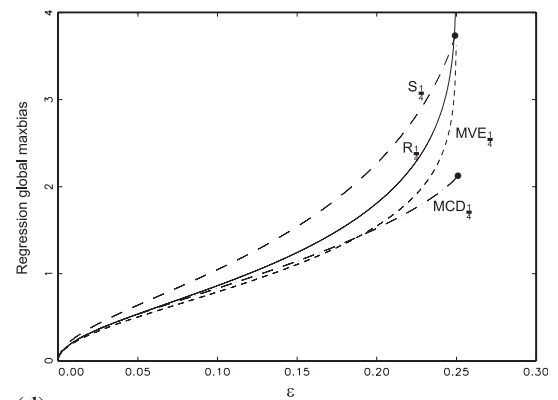

(d)

Fig. 1. Explosion maxbias (a), implosion maxbias (b), logarithmic global maxbias (c) and regression global maxbias (d) for the scale estimators $R_{\alpha}$ (plain line), $S_{\alpha}$ (dashed line), $\mathrm{MVE}_{\alpha}$ (shortdashed line) and $\mathrm{MCD}_{\alpha}$ (dashed-dotted line) at the Normal distribution for $\alpha=0.25$.

Even if the explosion maxbias curves are close together, the $\mathrm{MCD}_{\alpha}$ estimator is the most resistant to outliers. The explosion maxbias curves of both $S_{\alpha}$ and $\mathrm{MCD}_{\alpha}$ don't have a regular explosion and this makes them attractive with respect to other estimators with the same breakdown point. As far as robustness with respect to inliers is concerned, the MVE estimator outperforms all the others. The estimators $R_{\alpha}$ and $S_{\alpha}$ cannot compete with the $\mathrm{MVE}_{\alpha}$ and $\mathrm{MCD}_{\alpha}$ estimators when faced to inliers since they have a lower implosion breakdown point. Note that the implosion maxbias curves of the $\mathrm{MVE}_{\alpha}$ and $\mathrm{MCD}_{\alpha}$ estimators (for $\alpha<0.5$ ) do not even become zero when the percentage of contamination equals $50 \%$. For the global bias, the worst behavior is obtained by $S_{\alpha}$. The MCD estimator with $\alpha=0.25$ turns out to have the best overall behavior. From the regression global maxbias curve of $\mathrm{MCD}_{\alpha}$, it follows that the $\mathrm{LTS}_{\alpha}$ regression estimator for $\alpha=0.25$ has no regular explosion (and this remains true for all $\alpha<0.5$ ).

While the maxbias curve measures the robustness of an estimator, it is also important to know how precise an estimator is in the absence of outliers. Herefore, the asymptotic efficiencies, i.e. the relative asymptotic variance with respect to the maximum likelihood estimator, are computed at the Normal distribution (using results of Jurečková and Sen 1996, Grübel 1988, and Croux and Rousseeuw 1992). Table 1 reports the efficiencies of $R_{\alpha}, S_{\alpha}, \mathrm{MVE}_{\alpha}$ and $\mathrm{MCD}_{\alpha}$ for several values of $\alpha$. Some conclusions are that the efficiencies are decreasing in $\alpha(\geq 0.1)$, that $\mathrm{MVE}_{\alpha}$ and $\mathrm{MCD}_{\alpha}$ are more efficient than $R_{\alpha}$ and $S_{\alpha}$, and that $\alpha$-ranges are more efficient than $\alpha$-trimmed standard devia- 
Table 1. Efficiency of the scale estimators $R_{\alpha}, S_{\alpha}, \mathrm{MVE}_{\alpha}$ and $\mathrm{MCD}_{\alpha}$ for several values of $\alpha$ at the Normal model

\begin{tabular}{|c|cccccc|}
\cline { 2 - 7 } \multicolumn{1}{c|}{} & \multicolumn{6}{c|}{$\alpha$} \\
\hline Estimators & 0.1 & 0.2 & 0.25 & 0.3 & 0.4 & 0.5 \\
\hline$R_{\alpha}$ & 0.63 & 0.46 & 0.37 & 0.28 & 0.12 & - \\
$S_{\alpha}$ & 0.63 & 0.40 & 0.31 & 0.23 & 0.10 & - \\
$\mathrm{MVE}_{\alpha}$ & 0.64 & 0.63 & 0.60 & 0.56 & 0.46 & 0.37 \\
$\mathrm{MCD}_{\alpha}$ & 0.78 & 0.63 & 0.56 & 0.50 & 0.40 & 0.31 \\
\hline
\end{tabular}

Table 2. Optimal values of the summary measures $\gamma^{*}, \varepsilon^{*}$ and the efficiency Eff together with the associated choice of the parameter $\alpha$ for the scale estimators $R_{\alpha}, S_{\alpha}, \mathrm{MVE}_{\alpha}$ and $\mathrm{MCD}_{\alpha}$ at the Normal model. For every optimal choice, the values of the other (not optimized) summary values are also reported

\begin{tabular}{|c|cc|cc||cc|cc||cc|cc|}
\cline { 2 - 11 } \multicolumn{1}{c|}{} & \multicolumn{4}{c||}{ optimal $\gamma^{*}$} & \multicolumn{4}{c||}{ optimal $\varepsilon^{*}$} & \multicolumn{4}{c|}{ optimal Eff } \\
\hline Estimators & $\gamma^{*}$ & $\alpha$ & $\varepsilon^{*}$ & Eff & $\varepsilon^{*}$ & $\alpha$ & $\gamma^{*}$ & Eff & Eff & $\alpha$ & $\gamma^{*}$ & $\varepsilon^{*}$ \\
\hline$R_{\alpha}$ & 1.17 & 0.25 & 0.25 & 0.37 & 0.33 & 0.33 & 2.07 & 0.23 & 0.65 & 0.07 & 2.17 & 0.07 \\
$S_{\alpha}$ & 1.24 & 0.15 & 0.15 & 0.50 & 0.33 & 0.33 & 3.49 & 0.18 & 1 & 0 & $\infty$ & 0 \\
$\mathrm{MVE}_{\alpha}$ & 1.17 & 0.5 & 0.5 & 0.37 & 0.5 & 0.5 & 1.17 & 0.37 & 0.65 & 0.14 & 2.17 & 0.14 \\
$\mathrm{MCD}_{\alpha}$ & 1.24 & 0.3 & 0.3 & 0.50 & 0.5 & 0.5 & 2.09 & 0.31 & 1 & 0 & $\infty$ & 0 \\
\hline
\end{tabular}

tions while they are at the same time also more robust (for $\alpha$ not too small). Moreover, it appears that $\mathrm{MCD}_{0.25}$ is a bit less efficient than $\mathrm{MVE}_{0.25}$, while it is slightly more robust. The latter conclusion is somewhat surprising, since it is generally believed that MCD is more efficient and less robust than MVE. This may be true in the multivariate case (where $\mathrm{MVE}_{\alpha}$ even has non normal $n^{-1 / 2}$ convergence) but it is not confirmed in the univariate case. A recommendation for the best estimator to use will depend on $\alpha$ but the good performance of the MCD estimator at the Normal distribution has been pointed out.

Selection of the tuning parameter $\alpha$ is another subjective matter, which depends on the a priori belief in the percentage of outliers that could be present in the data. If one is sure that all observations follow the model, then efficiency is the only criterion to use. In a more exploratory data analysis context, it is customary to believe that 10 to $20 \%$ outliers may be expected (Huber 1981, page 3). Therefore, we will suppose that the realistic range of contamination is between 0 and $20 \%$ (a $25 \%$ breakdown estimator should be capable to cope with this amount of contamination). Table 2 reports the values of $\alpha$ which minimize the gross-error sensitivity $\gamma^{*}$ as local measure of robustness, optimize the breakdown point $\varepsilon^{*}$ as global measure of robustness, and the efficiency as measure of the precision for the four considered estimators at the Normal distribution. Since no single value of $\alpha$ will optimize the three measures at the same time, the values of the non optimized summary values are also given for every selected $\alpha$.

One sees from Table 2 that selecting $\alpha$ such that a high breakdown point, a high efficiency and a low gross-error sensitivity are combined is not easy. For the MVE estimator one could propose the choice $\alpha=0.5$, since it optimizes 


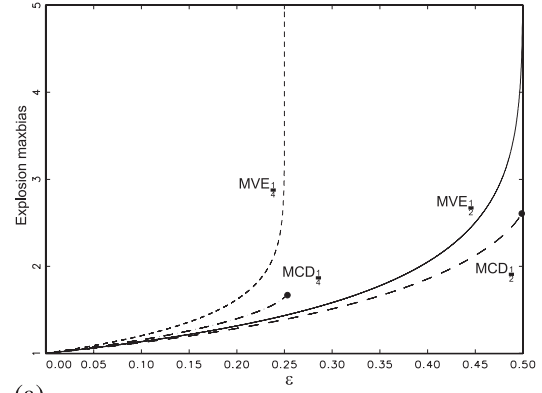

(a)

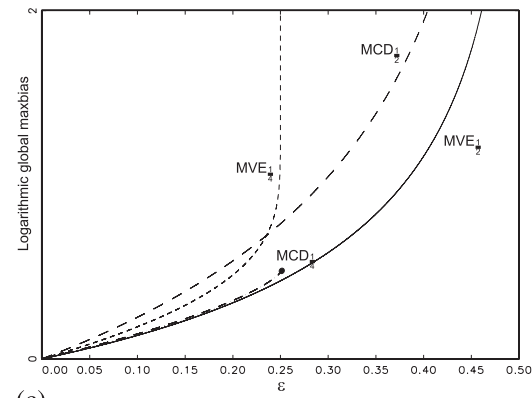

(c)

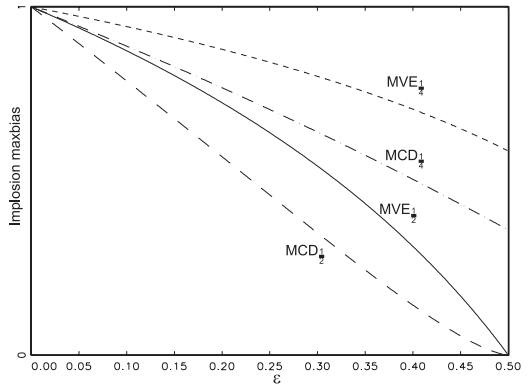

(b)

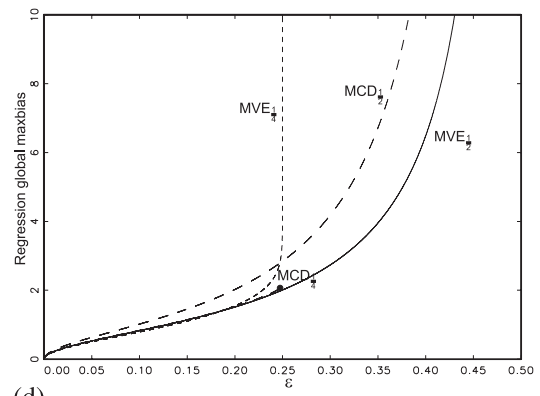

(d)

Fig. 2. Explosion maxbias (a), implosion maxbias (b), logarithmic global maxbias (c) and regression global maxbias (d) for the scale estimators $\mathrm{MVE}_{\alpha}$ (short-dashed line for $\alpha=0.25$ and plain line for $\alpha=0.5$ ) and $\mathrm{MCD}_{\alpha}$ (dashed-dotted line for $\alpha=0.25$ and dashed line for $\alpha=0.5$ ) at the Normal distribution.

two criteria $\left(\varepsilon^{*}\right.$ and $\left.\gamma^{*}\right)$. As $\alpha$-range estimator, the interquartile range seems to be a good compromise, while for the trimmed standard deviation $\alpha=0.20$ leads to a reasonable combination of robustness and efficiency. For the MCDscale estimator the choice $\alpha=0.25$ is defendable.

As a final comparison, Figure 2 gives the different maxbias curves of the $\mathrm{MVE}_{\alpha}$ and $\mathrm{MCD}_{\alpha}$ estimators at the Normal distribution for $\alpha=0.5$ and $\alpha=0.25$. The comparison between MCD and MVE is hard since the implosion maxbias is in favor of MVE while the explosion maxbias is in favor of MCD. The comparison gets even harder when one looks at the global maxbias curves. When $\alpha$ is small, $\mathrm{MCD}_{\alpha}$ has lower logarithmic and regression global maxbias curves than $\mathrm{MVE}_{\alpha}$. But for larger values of $\alpha \mathrm{MVE}_{\alpha}$ outperforms $\mathrm{MCD}_{\alpha}$, hereby confirming a minimax bias property of $\mathrm{LMS}_{\alpha}$ regression estimators (Yohai and Zamar 1993). Note however that the global maxbias of $\mathrm{MCD}_{1 / 4}$ is very close to the maxbias of $\mathrm{MVE}_{0.5}$ in the range of contamination of practical interest $[0,0.20]$.

\section{Conclusion}

The robustness of an estimator is most frequently measured by its breakdown point. However, even if two estimators have identical breakdown values, they may behave differently to given amounts of contamination. The maxbias 


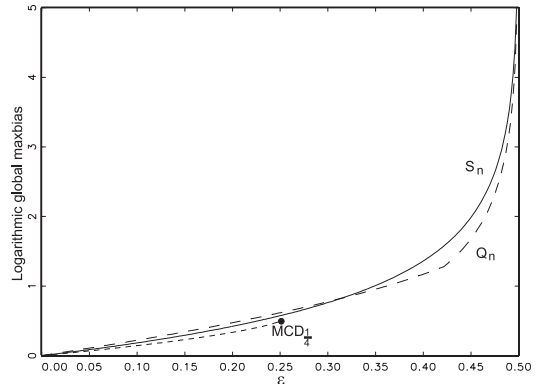

(a)

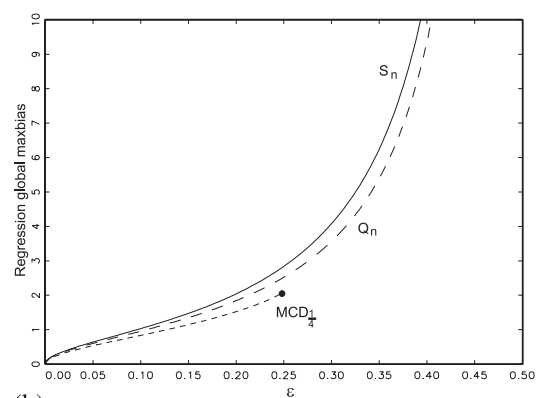

(b)

Fig. 3. Global maxbias curves of $S_{n}$ (plain line), $Q_{n}$ (dashed line) and $\mathrm{MCD}_{0.25}$ (short-dashed line) under the Normal model.

curve helps us to compare more thoroughly the robustness of the different estimators. In this paper, maxbias curves have been computed for some simple and frequently used univariate scale estimators: the $\alpha$-range, $\alpha$-trimmed standard deviation, $\mathrm{MVE}_{\alpha}$ and $\mathrm{MCD}_{\alpha}$ (which all have the same breakdown value for fixed $0<\alpha<1 / 3$ ).

The derivation of a maxbias curve of a scale estimator $S$ consists essentially in finding a worst contaminating distribution $H^{*}$ such that $S\left((1-\varepsilon) F+\varepsilon H^{*}\right)$ is maximal (or minimal for the implosion maxbias curve). It is tempting to conclude immediately that the worst bias is obtained by putting the contamination as far away as possible, so $H^{*}=\Delta_{\infty}$. Consider however the symmetric distribution $F=p_{1} \Delta_{-10}+\left(1-2 p_{1}\right) \Delta_{0}+p_{1} \Delta_{10}$ with $p_{1}=$ 0.101 , and the $\alpha$-range estimator $R_{\alpha}$ with $\alpha=10 \%$. It is easy to verify that $R_{\alpha}(F)=R_{\alpha}((1-\varepsilon) F+\varepsilon F)=20 c_{\alpha}>R_{\alpha}\left((1-\varepsilon) F+\varepsilon \Delta_{\infty}\right)=10 c_{\alpha}$, implying that $\Delta_{\infty}$ cannot be the worst contaminating distribution. The proofs in the appendix show however that, by adding the condition of unimodality on the model distribution, $H^{*}$ can still be taken as $\Delta_{\infty}$ (or more formally, as $\Delta_{x_{n}}$ for a big value of $n$ with $x_{n} \rightarrow \infty$ ) for the four considered scale functionals.

In Section 3, the $\mathrm{MCD}_{\alpha}$ scale estimator with $\alpha=0.25$ turned out to be a good compromise between robustness and efficiency. This remains of course the subjective viewpoint of the authors since no estimator will be optimal for all aspects (efficiency, gross-error sensitivity, breakdown point,...). Other scale estimators with explicit formulas, high breakdown point and good efficiency are the $S_{n}$ and $Q_{n}$ estimators proposed by Rousseeuw and Croux (1993). Figure 3 pictures the global maxbias curves of $S_{n}, Q_{n}$ and $\mathrm{MCD}_{1 / 4}$. It is seen that although the breakdown point of $S_{n}$ and $Q_{n}$ is $50 \%$, their maxbias is higher than the maxbias of $\mathrm{MCD}_{1 / 4}$ over the whole range $\left.] 0,0.20\right]$. When focus is not on maximal breakdown estimation, $\mathrm{MCD}_{1 / 4}$ appears to be a serious competitor for $S_{n}$ and $Q_{n}$. The loss of efficiency of $\mathrm{MCD}_{1 / 4}$ with respect to $Q_{n}(56 \%$ instead of $82 \%)$ remains however substantial.

The maxbias curves of the associated location estimators, the $\alpha$-midrange, the $\alpha$-trimmed mean and the location parts of the Minimum Volume Ellipsoid and Minimum Covariance Determinant estimators, have also been derived and can be found in Croux and Haesbroeck (1999b).

There still remains theoretical work to be done for the maxbias of multivariate location and scatter estimators. Yohai and Maronna (1990) computed 
maxbias curves for the multivariate MVE-scale estimator under the assumption of a fixed location while Adrover (1998) computed maxbias curves for Tyler's M-estimator. For the location MVE and MCD estimators however, no results seem to have been obtained.

\section{Appendix}

Proof of Proposition 1: Denote by $c^{+}$the right-hand side of (2.12) and let $\varepsilon<\alpha$.

First, let us show that $\sup _{G \in \mathscr{F}_{e}} R_{\alpha}(G) \geq c^{+}$. Consider the sequence of distribution functions $G_{n}=(1-\varepsilon) F+\varepsilon \Delta_{x_{n}}$ where $x_{n} \uparrow+\infty$. As soon as $x_{n}>$ $F^{-1}\left(\frac{1-\alpha}{1-\varepsilon}\right)$, one obtains that $G_{n}^{-1}(1-\alpha)=F^{-1}\left(\frac{1-\alpha}{1-\varepsilon}\right)$ and $G_{n}^{-1}(\alpha)=$ $F^{-1}\left(\frac{\alpha}{1-\varepsilon}\right)$ for $\varepsilon<\alpha$, which yields $\lim _{n \rightarrow+\infty} R_{\alpha}\left(G_{n}\right)=c^{+}$.

Now, let $H$ be an arbitrary distribution function and let us show that $R_{\alpha}(G) \leq c^{+}$where $G=(1-\varepsilon) F+\varepsilon H$. By Lemma 2, there exists $\varepsilon_{1}$ with $0 \leq \varepsilon_{1} \leq \varepsilon$, such that

$$
R_{\alpha}(G) \leq c_{\alpha}\left(F^{-1}\left(\frac{1-\alpha-\varepsilon_{1}}{1-\varepsilon}\right)-F^{-1}\left(\frac{\alpha-\varepsilon_{1}}{1-\varepsilon}\right)\right):=g\left(\varepsilon_{1}\right) .
$$

Moreover, by the unimodality condition on $F$, the function $g\left(\varepsilon_{1}\right)$, for $0 \leq \varepsilon_{1} \leq \varepsilon$ is symmetric about $\frac{\varepsilon}{2}$, which is also its minimum, while $\varepsilon_{1}=0$ or $\varepsilon$ both lead to the maximum value $c^{+}$for $g\left(\varepsilon_{1}\right)$. Therefore, $R_{\alpha}(G) \leq c^{+}$for any distribution $G$.

If $\alpha \leq \varepsilon \leq 1-\alpha$, the contaminated distribution $G_{n}$ leads to an infinite bias for the functional $R_{\alpha}$. By taking $x_{n}>\left|F^{-1}\left(\frac{1-\alpha-\varepsilon}{1-\varepsilon}\right)\right|$, it follows that $\quad G_{n}^{-1}(1-\alpha)=x_{n} \quad$ while $\quad G_{n}^{-1}(\alpha)=F^{-1}\left(\frac{\alpha}{1-\varepsilon}\right)$. So, $\quad R_{\alpha}\left(G_{n}\right)=$ $c_{\alpha}\left(x_{n}-F^{-1}\left(\frac{\alpha}{1-\varepsilon}\right)\right)$ which goes to infinity as $n$ increases.

For the implosion maxbias curve, denote by $c^{-}$the right-hand side of (2.13) and consider a contamination percentage $\varepsilon<1-2 \alpha$. If $G=(1-\varepsilon) F+$ $\varepsilon \Delta_{0}$, then $R_{\alpha}(G)=c^{-}$since in that case, $G^{-1}(\alpha)=F^{-1}\left(\frac{\alpha}{1-\varepsilon}\right)$ and $G^{-1}(1-\alpha)$ $=F^{-1}\left(\frac{1-\alpha-\varepsilon}{1-\varepsilon}\right)$. Now, if $G=(1-\varepsilon) F+\varepsilon H$ where $H$ is any distribution, then $R_{\alpha}(G) \geq c^{-}$since $G^{-1}(\alpha) \leq F^{-1}\left(\frac{\alpha}{1-\varepsilon}\right)$ and $G^{-1}(1-\alpha) \geq F^{-1}\left(\frac{1-\alpha-\varepsilon}{1-\varepsilon}\right)$. The proof is completed when we note that $G^{-1}(1-\alpha)=G^{-1}(\alpha)=0$ for $H=\Delta_{0}$ whenever $\varepsilon \geq 1-2 \alpha$.

Proof of Proposition 2: Denote by $c^{+}$the right-hand side of (2.14) and let $\varepsilon<\alpha$. First, consider a sequence of distribution functions $G_{n}=$ 
$(1-\varepsilon) F+\varepsilon \Delta_{x_{n}}$ where $x_{n} \uparrow+\infty$. We claim that $\lim _{n \rightarrow+\infty} S_{\alpha}^{2}\left(G_{n}\right)=c^{+}$. By taking $x_{n}>F^{-1}\left(\frac{1-\alpha}{1-\varepsilon}\right), G_{n}^{-1}(t)=F^{-1}\left(\frac{t}{1-\varepsilon}\right)$ for all $0<t \leq 1-\alpha$. So, when $x_{n}>F^{-1}\left(\frac{1-\alpha}{1-\varepsilon}\right)$, with definition $(2.11)$ of $S_{\alpha}^{2}$,

$$
\begin{aligned}
S_{\alpha}^{2}\left(G_{n}\right) & =\frac{d_{\alpha}}{1-2 \alpha} \int_{\alpha}^{1-\alpha}\left(G_{n}^{-1}(t)-T_{\alpha}\left(G_{n}\right)\right)^{2} d t \\
& =\frac{d_{\alpha}}{1-2 \alpha} \int_{\alpha}^{1-\alpha}\left(F^{-1}\left(\frac{t}{1-\varepsilon}\right)-T_{\alpha}\left(G_{n}\right)\right)^{2} d t
\end{aligned}
$$

where $T_{\alpha}\left(G_{n}\right)=\frac{1}{1-2 \alpha} \int_{\alpha}^{1-\alpha} G_{n}^{-1}(t) d t=\frac{1}{1-2 \alpha} \int_{\alpha}^{1-\alpha} G^{-1}\left(\frac{t}{1-\varepsilon}\right) d t=\tilde{t} . \quad$ By transforming the variable $t$ to $u=t /(1-\varepsilon)$ we get $S_{\alpha}^{2}\left(G_{n}\right)=c^{+}$.

Now, let $G=(1-\varepsilon) F+\varepsilon H$ where $H$ is an arbitrary distribution function. By Lemma $2(b)$, there exists $\varepsilon_{1} \in[0, \varepsilon]$ such that

$$
S_{\alpha}^{2}(G) \leq \frac{d_{\alpha}(1-\varepsilon)}{1-2 \alpha} \int_{q_{1}\left(\varepsilon_{1}, \alpha\right)}^{q_{2}\left(\varepsilon_{1}, \alpha\right)}\left(y-t\left(\varepsilon_{1}\right)\right)^{2} d F(y):=h\left(\varepsilon_{1}\right),
$$

where $t\left(\varepsilon_{1}\right)=\frac{1-\varepsilon}{1-2 \alpha} \int_{q_{1}\left(\varepsilon_{1}, \alpha\right)}^{q_{2}\left(\varepsilon_{1}, \alpha\right)} y d F(y), q_{1}\left(\varepsilon_{1}, \alpha\right)=F^{-1}\left(\frac{\alpha-\varepsilon_{1}}{1-\varepsilon}\right)$ and $q_{2}\left(\varepsilon_{1}, \alpha\right)=$ $F^{-1}\left(\frac{1-\alpha-\varepsilon_{1}}{1-\varepsilon}\right)$. The function $h\left(\varepsilon_{1}\right)$, for $0 \leq \varepsilon_{1} \leq \varepsilon$, is symmetric about $\frac{\varepsilon}{2}$ and its derivative equals

$$
h^{\prime}\left(\varepsilon_{1}\right)=\frac{1}{1-2 \alpha}\left(q_{2}\left(\varepsilon_{1}, \alpha\right)-q_{1}\left(\varepsilon_{1}, \alpha\right)\right)\left(2 t\left(\varepsilon_{1}\right)-q_{1}\left(\varepsilon_{1}, \alpha\right)-q_{2}\left(\varepsilon_{1}, \alpha\right)\right) .
$$

The sign of $h^{\prime}\left(\varepsilon_{1}\right)$ is the sign of $2 t\left(\varepsilon_{1}\right)-q_{1}\left(\varepsilon_{1}, \alpha\right)-q_{2}\left(\varepsilon_{1}, \alpha\right)$. For $\varepsilon_{1}>\frac{\varepsilon}{2}$, $q_{1}\left(\varepsilon_{1}, \alpha\right)+q_{2}\left(\varepsilon_{1}, \alpha\right)<0$ and

$$
t\left(\varepsilon_{1}\right)=\frac{\int_{q_{1}\left(\varepsilon_{1}, \alpha\right)}^{q_{2}\left(\varepsilon_{1}, \alpha\right)} y d F(y)}{P_{F}\left(q_{1}\left(\varepsilon_{1}, \alpha\right)<Y<q_{2}\left(\varepsilon_{1}, \alpha\right)\right)} \geq \frac{1}{2}\left(q_{1}\left(\varepsilon_{1}, \alpha\right)+q_{2}\left(\varepsilon_{1}, \alpha\right)\right)
$$

by unimodality of $F$. Therefore, $\varepsilon_{1}=\frac{\varepsilon}{2}$ gives the minimum value for $h\left(\varepsilon_{1}\right)$ while the maximum is attained for $\varepsilon_{1}=0$. Thus, $S_{\alpha}^{2}(G) \leq h\left(\varepsilon_{1}\right) \leq h(0)=c^{+}$.

If $\alpha<\varepsilon(\leq 1-\alpha)$, the contaminated distribution $G_{n}$ will lead to an infinite bias for the functional $S_{\alpha}$ when $x_{n}>\left|F^{-1}\left(\frac{1-\alpha-\varepsilon}{1-\varepsilon}\right)\right|$. Then $G_{n}^{-1}(1-\alpha)=$ $x_{n}$ while $F_{n, \varepsilon}^{-1}(t)=F^{-1}\left(\frac{t}{1-\varepsilon}\right)$ for all $\alpha \leq t \leq \frac{1}{2}$. So, the following inequalities may be deduced 


$$
\begin{aligned}
S_{\alpha}^{2}\left(G_{n}\right) & =\frac{d_{\alpha}}{1-2 \alpha} \int_{\alpha}^{1-\alpha}\left(G_{n}^{-1}(t)-T_{\alpha}\left(G_{n}\right)\right)^{2} d t \\
& \geq \frac{d_{\alpha}}{1-2 \alpha} \int_{\alpha}^{1 / 2}\left(F^{-1}\left(\frac{t}{1-\varepsilon}\right)-T_{\alpha}\left(G_{n}\right)\right)^{2} d t .
\end{aligned}
$$

Now, for any $x_{n}>F^{-1}\left(\frac{1-\alpha-\varepsilon}{1-\varepsilon}\right)$, the functional $T_{\alpha}$ as defined by $(2.8)$ is given by

$$
T_{\alpha}\left(G_{n}\right)=\frac{1}{1-2 \alpha}\left\{(1-\varepsilon) \int_{F^{-1}(\alpha /(1-\varepsilon))}^{x_{n}} y d F(y)+\left(1-\alpha-(1-\varepsilon) F\left(x_{n}\right)\right) x_{n}\right\},
$$

since $\delta_{1}\left(G_{n}\right)=0$. When $\varepsilon>\alpha$,

$$
\lim _{n \rightarrow+\infty} T_{\alpha}\left(G_{n}\right)=\frac{1}{1-2 \alpha}\left\{(1-\varepsilon) \int_{F^{-1}(\alpha /(1-\varepsilon))}^{+\infty} y d F(y)+(\varepsilon-\alpha) \lim _{n \rightarrow \infty} x_{n}\right\}=\infty .
$$

This yields $\lim _{n \rightarrow+\infty} S_{\alpha}^{2}\left(G_{n}\right)=\infty$.

For the implosion maxbias curve, denote by $c^{-}$the right-hand side of (2.15) and consider first a contamination less than $1-2 \alpha$. First, we show that if $G_{0}=(1-\varepsilon) F+\varepsilon \Delta_{0}$, then $S_{\alpha}^{2}\left(G_{0}\right)=c^{-}$. When $\varepsilon<1-2 \alpha, G_{0}^{-1}(\alpha)=$ $F^{-1}\left(\frac{\alpha}{1-\varepsilon}\right), G_{0}^{-1}(1-\alpha)=F^{-1}\left(\frac{1-\alpha-\varepsilon}{1-\varepsilon}\right)$ and $\delta_{1}\left(G_{0}\right)=\delta_{2}\left(G_{0}\right)=0$. It is easy to verify now, using the symmetry of $F$, that $T_{\alpha}\left(G_{0}\right)=0$ and $S_{\alpha}\left(G_{0}\right)=c^{-}$.

Now, if $G=(1-\varepsilon) F+\varepsilon H$ where $H$ can be any distribution, then by definition $(2.9)$ of $S_{\alpha}^{2}(G)$,

$$
\begin{aligned}
S_{\alpha}^{2}(G) & \geq \frac{d_{\alpha}}{1-2 \alpha} \int_{G^{-1}(\alpha)}^{G^{-1}(1-\alpha)}\left(y-T_{\alpha}(G)\right)^{2} d G(y) \\
& \geq \frac{d_{\alpha}(1-\varepsilon)}{1-2 \alpha} \int_{G^{-1}(\alpha)}^{G^{-1}(1-\alpha)}\left(y-T_{\alpha}(G)\right)^{2} d F(y) .
\end{aligned}
$$

Using the inequalities $G^{-1}(\alpha) \leq F^{-1}\left(\frac{\alpha}{1-\varepsilon}\right)$ and $G^{-1}(1-\alpha) \geq F^{-1}\left(\frac{1-\alpha-\varepsilon}{1-\varepsilon}\right)$, one gets

$$
\begin{aligned}
S_{\alpha}^{2}(G) & \geq \frac{d_{\alpha}(1-\varepsilon)}{1-2 \alpha} \int_{F^{-1}(\alpha /(1-\varepsilon))}^{F^{-1}((1-\alpha-\varepsilon) /(1-\varepsilon))}\left(t-T_{\alpha}(G)\right)^{2} d F \\
& =\frac{d_{\alpha}(1-\varepsilon)}{1-2 \alpha}\left\{\int_{F^{-1}(\alpha /(1-\varepsilon))}^{F^{-1}((1-\alpha-\varepsilon) /(1-\varepsilon))} t^{2} d F(t)+\frac{1-2 \alpha-\varepsilon}{1-\varepsilon} T_{\alpha}^{2}(G)\right\} \geq\left(c^{-}\right)^{2} .
\end{aligned}
$$

The proof is completed by noting that, for $\varepsilon \geq 1-2 \alpha, G^{-1}(\alpha)=$ $G^{-1}(1-\alpha)=0$ for $H=\Delta_{0}$ yielding implosion to zero of $S_{\alpha}$. 
Proof of Proposition 4: Let $c^{+}$denote the right-hand side of (2.28) and let $\varepsilon<\alpha$. First it is shown that $\operatorname{MCD}_{\alpha}(G) \leq\left(c^{+}\right)^{2}$ where $G=(1-\varepsilon) F+\varepsilon H$ with $H$ arbitrary. By definition, $\operatorname{MCD}_{\alpha}(G) \leq \lambda_{G}(0)$ with

$$
\begin{aligned}
\lambda_{G}(0) & =\frac{1}{1-\alpha}\left(\int_{-H_{G}(0)}^{H_{G}(0)} y^{2} d G(x)+\delta_{G}(0) H_{G}(0)^{2}\right) \\
& =\frac{1}{1-\alpha}\left((1-\varepsilon) \int_{-H_{G}(0)}^{H_{G}(0)} y^{2} d F(x)+\varepsilon \int_{-H_{G}(0)}^{H_{G}(0)} y^{2} d H(x)+\delta_{G}(0) H_{G}(0)^{2}\right) .
\end{aligned}
$$

Now, $0 \leq H_{G}(0) \leq H_{\varepsilon}^{+}(0)$ (since $(1-\varepsilon) P_{F}\left(-H_{G}(0) \leq X \leq H_{G}(0)\right) \leq 1-\alpha$ ) and (5.1) can be rewritten as

$$
\lambda_{G}(0)=\frac{1}{1-\alpha}\left((1-\varepsilon) \int_{-H_{\varepsilon}^{+}(0)}^{H_{\varepsilon}^{+}(0)} y^{2} d F(x)+I_{1}+I_{2}\right),
$$

with

$$
\begin{aligned}
& I_{1}=\varepsilon \int_{-H_{G}(0)}^{H_{G}(0)} y^{2} d H(x)+\delta_{G}(0) H_{G}(0)^{2} \\
& I_{2}=-(1-\varepsilon)\left(\int_{-H_{\varepsilon}^{+}(0)}^{-H_{G}(0)} y^{2} d F(x)+\int_{H_{G}(0)}^{H_{\varepsilon}^{+}(0)} y^{2} d F(x)\right) .
\end{aligned}
$$

Now

$$
\begin{aligned}
& I_{1} \leq H_{G}^{2}(0)\left(\varepsilon P_{H}\left(|X|<H_{G}(0)\right)+\delta_{G}(0)\right) \\
& I_{2} \leq-2(1-\varepsilon) H_{G}(0)^{2} \int_{H_{G}(0)}^{-H_{\varepsilon}^{+}(0)} d F(x)
\end{aligned}
$$

and

$$
\begin{aligned}
2(1-\varepsilon) \int_{H_{G}(0)}^{H_{\varepsilon}^{+}(0)} d F(x) \\
\quad=(1-\varepsilon) P_{F}\left(H_{G}(0)<|X|<H_{\varepsilon}^{+}(0)\right) \\
=(1-\varepsilon)\left(P_{F}\left(|X|<H_{\varepsilon}^{+}(0)\right)-P_{F}\left(|X|<H_{G}(0)\right)\right) \\
\quad=(1-\varepsilon)\left(\frac{1-\alpha}{1-\varepsilon}-P_{F}\left(|X|<H_{G}(0)\right)\right) \\
=(1-\alpha)-(1-\varepsilon) P_{F}\left(|X|<H_{G}(0)\right)=\varepsilon P_{H}\left(|X|<H_{G}(0)\right)+\delta_{G}(0),
\end{aligned}
$$

yielding $I_{1}+I_{2} \leq 0$. Therefore, (5.2) gives $\operatorname{MCD}_{\alpha}^{2}(G) \leq \lambda_{G}(0) \leq\left(c^{+}\right)^{2}$.

Let us now consider the sequence of distribution functions $G_{n}=$ $(1-\varepsilon) F+\varepsilon \Delta_{x_{n}}$ with $x_{n} \uparrow+\infty$. Denote by $y_{n}$ the solution of the equation 
$y+H_{\varepsilon}^{+}(y)=x_{n}$ and $z_{n}$ the solution of $z+H_{\varepsilon}^{-}(z)=x_{n}$. It immediately follows that $y_{n}<z_{n}<x_{n}$. Since $\varepsilon<\alpha, y_{n}>0$ for $n$ sufficiently large. Moreover, it follows from the definitions of $z_{n}$ and $H_{\varepsilon}^{-}(z)$ that

$$
\begin{aligned}
F\left(x_{n}\right)-F\left(2 z_{n}-x_{n}\right) & =\frac{1-\alpha-\varepsilon}{1-\varepsilon} \\
& \Leftrightarrow z_{n}=\frac{1}{2}\left(x_{n}+F^{-1}\left(F\left(x_{n}\right)-\frac{1-\alpha-\varepsilon}{1-\varepsilon}\right)\right)
\end{aligned}
$$

which shows that $z_{n} \rightarrow+\infty$ as $n \rightarrow+\infty$. Also, $x_{n}-z_{n}$ tends to infinity, otherwise $2 z_{n}-x_{n}$ would tend to infinity implying that $\int_{2 z_{n}-x_{n}}^{x_{n}} d F(y) \rightarrow 0$, contradicting the above equation.

By definition, $\operatorname{MCD}_{\alpha}^{2}\left(G_{n}\right)=\min _{x \in \mathbb{R}} \lambda_{G_{n}}(x)$, but by symmetry, one may restrict the values of $x$ to $[0,+\infty)$. Let us partition this interval into three parts: $\left.\left.\left[0, y_{n}\right],\right] y_{n}, z_{n}\right]$ and $\left.] z_{n},+\infty\right)$.

1. For $x \leq y_{n}$, we have $H_{G_{n}}(x)=H_{\varepsilon}^{+}(x)$ and

$$
\begin{aligned}
\lambda_{G_{n}}(x) & =\frac{(1-\varepsilon)}{1-\alpha} \int_{x-H_{\varepsilon}^{+}(x)}^{x+H_{\varepsilon}^{+}(x)}(y-x)^{2} d F(y) \\
& \geq \frac{l_{\alpha}(1-\varepsilon)}{1-\alpha} \int_{-H_{\varepsilon}^{+}(0)}^{H_{\varepsilon}^{+}(0)} y^{2} d F(y)=\lambda_{G_{n}}(0),
\end{aligned}
$$

by Lemma 3.

2. For $y_{n}<x \leq z_{n}$,

$$
\lambda_{G_{n}}(x)=\frac{1-\varepsilon}{1-\alpha} \int_{2 x-x_{n}}^{x_{n}}(y-x)^{2} d F(y)+\frac{\delta_{G_{n}}(x)\left(x_{n}-x\right)^{2}}{1-\alpha},
$$

where $\delta_{G_{n}}(x)>0$ and $\left|x-x_{n}\right| \geq\left|z_{n}-x_{n}\right|$. For $n$ sufficiently large, it holds therefore that $\lambda_{G_{n}}(x) \geq \lambda_{G_{n}}(0)$ since $\left|z_{n}-x_{n}\right| \rightarrow+\infty$.

3. For $z_{n}<x \leq x_{n}$ (as well as for $x>x_{n}$ ),

$$
\lambda_{G_{n}}(x)=\frac{(1-\varepsilon)}{1-\alpha} \int_{x-H_{\varepsilon}^{-}(x)}^{x+H_{\varepsilon}^{-}(x)}(y-x)^{2} d F(y)+\frac{\varepsilon\left(x-x_{n}\right)^{2}}{1-\alpha},
$$

where the integral tends to infinity when $n \rightarrow+\infty$ by Lemma 3 .

We conclude that

$$
\begin{aligned}
\operatorname{MCD}_{\alpha}^{2}\left(G_{n}\right) & =l_{\alpha} \min _{x} \lambda_{G_{n}}(x)=l_{\alpha} \lambda_{G_{n}}(0) \\
& =\frac{l_{\alpha}(1-\varepsilon)}{1-\alpha} \int_{-H_{\varepsilon}^{+}(0)}^{H_{\varepsilon}^{+}(0)} y^{2} d F(y)=\left(c^{+}\right)^{2},
\end{aligned}
$$

when $\varepsilon<\alpha$. 
For $\varepsilon>\alpha$ the same reasoning can be followed to prove that $G_{n}$ leads to an infinite bias. The optimal interval always contains the contaminating mass (cases 2 and 3) and at least one of the two terms in $\lambda_{G_{n}}(x)$ goes to infinity as $n$ increases.

For the implosion maxbias curve, denote by $c^{-}$the right-hand side of (2.29) and let us show that $\operatorname{MCD}_{\alpha}^{2}\left(G_{0}\right)=\left(c^{-}\right)^{2}$ for $G_{0}=(1-\varepsilon) F+\varepsilon \Delta_{0}$ when $\varepsilon<1-\alpha$. For any $x$, we have

$$
\begin{aligned}
\lambda_{G_{0}}(x) & \geq \frac{1-\varepsilon}{1-\alpha} \int_{x-H_{G_{0}}(x)}^{x+H_{G_{0}}(x)}(y-x)^{2} d F(y) \\
& \geq \frac{1-\varepsilon}{1-\alpha} \int_{-H_{G_{0}}(x)}^{H_{G_{0}}(x)} y^{2} d F(y) \\
& \geq \frac{1-\varepsilon}{1-\alpha} \int_{-H_{G_{0}}(0)}^{H_{G_{0}}(0)} y^{2} d F(y)=\lambda_{G}(0)
\end{aligned}
$$

using first the unimodality of $F$ and then the inequality $H_{G_{0}}(x) \geq H_{G_{0}}(0)$. Therefore, $\operatorname{MCD}_{\alpha}^{2}\left(G_{0}\right)=l_{\alpha} \lambda_{G_{0}}(0)=\left(c^{-}\right)^{2}$ since $\operatorname{MCD}_{\alpha}^{2}$ is the minimum of $\lambda_{G_{0}}(x)$ and $H_{G_{0}}(0)=H_{\varepsilon}^{-}(0)$.

Now, for an arbitrary distribution $H$, let us prove that $\operatorname{MCD}_{\alpha}(G) \geq c^{-}$ with $G=(1-\varepsilon) F+\varepsilon H$. Let $T(G)$ denote the MCD location estimate, that is $T(G)=\operatorname{argmin}_{x} \lambda_{G}(x)$. Since $P_{G}\left(|X-T(G)| \leq H_{G}(T(G))\right) \geq 1-\alpha$, we have

$$
\begin{aligned}
(1-\varepsilon) P_{F}(|X-T(G)| & \left.\leq H_{G}(T(G))\right)+\varepsilon \geq 1-\alpha \\
& \Leftrightarrow P_{F}\left(|X-T(G)| \leq H_{G}(T(G))\right) \geq \frac{1-\alpha-\varepsilon}{1-\varepsilon},
\end{aligned}
$$

yielding $H_{G}(T(G)) \geq H_{\varepsilon}^{-}(T(G))$. Therefore

$$
\begin{aligned}
\operatorname{MCD}_{\alpha}^{2}(G)=l_{\alpha} \lambda_{G}(T(G)) & \geq \frac{l_{\alpha}(1-\varepsilon)}{1-\alpha} \int_{T(G)-H_{\varepsilon}^{-}(T(G))}^{T(G)+H_{\varepsilon}^{-}(T(G))}(y-T(G))^{2} d F(y) \\
& \geq \frac{l_{\alpha}(1-\varepsilon)}{1-\alpha} \int_{-H_{\varepsilon}^{-}(0)}^{H_{\varepsilon}^{-}(0)} y^{2} d F(y),
\end{aligned}
$$

by Lemma 3 .

If $\varepsilon \geq 1-\alpha$, then the distribution $G_{0}=(1-\varepsilon) F+\varepsilon \Delta_{0}$ satisfies $\operatorname{MCD}_{\alpha}\left(G_{0}\right)$ $=0$ since $\lambda_{G}(0)=0$, causing implosion of the scale functional.

\section{References}

Adrover JG (1998) Minimax bias-robust estimation of the dispersion matrix of a multivariate distribution. The Annals of Statistics 26:2301-2320

Berrendero JR, Mazzi S, Romo J, Zamar RH (1998) On the explosion rate of maximum bias functions. Canadian Journal of Statistics 26:333-351 
Berrendero JR, Romo J (1998) Stability under contamination of robust regression estimators based on differences of residuals. Journal of Statistical Planning and Inference 70:149-165

Berrendero JR, Zamar, RH (1999) Global robustness of location and dispersion estimates. Statistics and Probability Letters 44:63-72

Bickel PJ, Lehmann EL (1976) Descriptive statistics for nonparametric models. III. Dispersion. The Annals of Statisticsc 4:1139-1159

Butler RW, Davies PL, Jhun M (1993) Asymptotics for the minimum covariance determinant estimator. The Annals of Statistics 21:1385-1400

Croux C, Haesbroeck G (1999a) Influence function and efficiency of the minimum covariance determinant scatter matrix estimator. Journal of Multivariate Analysis 71:161-190

Croux C, Haesbroeck G (1999b) Maxbias curves of robust scale estimators based on subranges. Technical Report. University of Liège: http://www.eaa.egss.ulg.ac.be/Haesbroeck

Croux C, Rousseeuw PJ (1992) A class of high-breakdown scale estimators based on subranges. Communications in Statistics-Theory and Methods 21:1935-1951

Croux C, Rousseeuw PJ, Van Bael A (1996) Positive breakdown regression by minimizing nested scale estimators. Journal of Statistical Planning and Inference 53:197-235

Grübel R (1988) The length of the shorth. The Annals of Statistics 16:619-628

Hampel FR, Ronchetti EM, Rousseeuw PJ, Stahel WA (1986) Robust statistics: The approach based on influence functions. Wiley, New York

He X, Simpson DG (1993) Lower bounds for contamination bias: Globally minimax versus locally linear estimation. The Annals of Statistics 21:314-337

Hössjer O, Rousseeuw PJ, Croux C (1996) Asymptotics of an estimator of a robust spread functional. Statistica Sinica 6:375-388

Huber PJ (1981) Robust statistics. Wiley, New York

Jurečová J, Sen PK (1996) Robust statistical procedures, asymptotics and interrelations. Wiley, New York

Martin RD, Yohai VJ, Zamar RH (1989) Min-max bias robust regression. The Annals of Statistics 17:1608-1630

Martin RD, Zamar RH (1989) Asymptotically min-max bias robust $M$-estimates of scale for positive random variables. Journal of the American Statistical Association 84:494-501

Martin RD, Zamar RH (1993) Bias robust estimation of scale. The Annals of Statistics 21:9911017

Rousseeuw PJ (1985) Multivariate estimation with high breakdown point. In: Grossmann W, Pflug G, Vincze I, Wertz W (eds.) Mathematical Statistics and Applications (Vol. B). Reidel, Dordrecht, pp. 283-297

Rousseeuw PJ (1998) Maxbias curves. In: Kotz S, Read C, Banks D (eds.) Encyclopedia of Statistical Sciences Update Volume 3. Wiley, New York

Rousseeuw PJ, Croux C (1993) Alternatives to the median absolute deviation. Journal of the American Statistical Association 88:1273-1283

Rousseeuw PJ, Leroy AM (1987) Robust regression and outlier detection. Wiley, New York

Rousseeuw PJ, Leroy AM (1988) A robust scale estimator based on the shortest half. Statistica Neerlandica 42:103-116

Yohai VJ, Maronna RA (1990) The maximum bias of robust covariances. Communications in Statistics Part A - Theory and Methods 19:3925-3933

Yohai VJ, Zamar RH (1993) A minimax-bias property of the least $\alpha$-quantile estimates. The Annals of Statistics 4:1824-1842 